\title{
LA NUEVA PRESTACIÓN NO CONTRIBUTIVA: EL INGRESO MÍNIMO VITAL
}

\author{
Ana Nieves Escribá Pérez \\ Directora del Grado en Derecho \\ Profesora de Derecho del Trabajo y de la Seguridad Social \\ Universidad Internacional de Valencia.
}

\begin{abstract}
El Ingreso Mínimo Vital está claramente fundamentado en los pilares del Estado social y democrático de derecho y, por supuesto, en el carácter eminentemente social de nuestro tipo de Estado. Es obligación de los poderes públicos garantizar el bienestar de todos y cada uno de los miembros de nuestra sociedad y dentro de dicho ámbito debe enmarcarse la nueva prestación no contributiva de nuestro sistema de seguridad social. Basada en el principio de igualdad y, por ende, en el de universalidad -concebidos como dos pilares básicos del ordenamiento jurídico de la seguridad social-, la nueva prestación tiene como finalidad la erradicación de la pobreza -en la medida de lo posible- y la protección de los colectivos más desfavorecidos y, por consiguiente, la inclusión de los mismos tanto en la vida social como en la laboral. Una prestación que se hacía necesaria y que se ha visto materializada de forma abrupta debido a la irrupción de la COVID-19 en nuestra sociedad. Este artículo trata de esclarecer las cuestiones técnicas de la nueva norma, así como el cumplimiento, por parte de los poderes públicos, de la obligación de velar por el bienestar de la ciudadanía.
\end{abstract}

The Minimum Vital Income is clearly based on the pillars of the social and democratic State of law and, of course, on the eminently social character of our type of State. It is the obligation of the public powers to guarantee the well-being of each and every one of the members of our society and within this scope the new non-contributory benefit of our social security system must be framed. Based on the principle of equality and, therefore, on universality -conceived as two basic pillars of the social security legal system-, the new benefit is aimed at eradicating poverty -to the extent possible-and the protection of the most disadvantaged groups and, consequently, the inclusion of these groups in both social and work life. A provision that was necessary and that has been materialized abruptly due to the irruption of COVID-19 in our society. This article tries to clarify the technical issues of the new norm as well as the fulfillment, by the public powers, of the obligation to ensure the well-being of the citizens.

IUSLabor 1/2021, ISSN 1699-2938, p. 98-123

DOI. 10.31009/IUSLabor.2021.101.04 
Tittle: The new non-contributive benefit: the minimum vital income

Palabras clave: Prestación no contributiva, Ingreso Mínimo Vital, Seguridad Social. Key words: No-Contributive Benefit, Minimum Vital Income, Social Security.

Fecha envío: 08.10.2020 | Fecha aceptación: 02.02.2021

\section{Sumario}

1. Introducción

2. El sistema de seguridad social español y las prestaciones no contributivas

3. El carácter de prestación no contributiva del Ingreso Mínimo Vital

4. El Ingreso Mínimo Vital, en síntesis

4.1 Conceptos

a. Ingreso Mínimo Vital

b. Unidad de Convivencia

c. Vulnerabilidad Económica

4.2 Requisitos y Características

4.3 Plazo de presentación

4.4 Duración

4.5 Solicitud

4.6 Reconocimiento y control

4.7 Obligaciones, Infracciones y Sanciones

4.8 Cuantía

5. El Ingreso Mínimo Vital: Compatibilidades e incompatibilidades entre prestaciones

6. El carácter universal del Ingreso Mínimo Vital

7. Conclusiones

8. Bibliografía 


\section{Introducción}

La finalidad del Ingreso Mínimo Vital -en adelante IMV- está fundamentada en el carácter social de nuestro sistema de seguridad social que garantiza, entre otras cuestiones, la intervención del Estado en situaciones de especial vulnerabilidad; en el caso de esta nueva prestación se pretende proteger una situación de necesidad basada en la falta de ingresos o el carácter mínimo de los estos que conducen a que, en ocasiones, esta roce el umbral de la pobreza y, es por ello que, el Estado, debe tratar de garantizar la subsistencia y el bienestar de ciertos colectivos. Esta prestación es, sin lugar a dudas, una herramienta cuyo propósito es evitar la exclusión social de aquellos colectivos de personas más vulnerables y con menos posibilidades que nace con la pretensión inequívoca de garantizar la igualdad de oportunidades que, sin lugar a dudas, se traducirá en un acercamiento a la igualdad efectiva en materia económica en la sociedad actual ${ }^{1}$.

A tenor del artículo 148.1.20 de la Constitución Española -en adelante CE- las Comunidades Autónomas -en adelante CCAA- se han hecho responsables de establecer y gestionar cualquier cuestión referente a políticas de rentas mínimas lo que suponía que cada una de ellas establecía los caracteres y el desarrollo de las mismas y, por tanto, se traducía en que una persona recibiría o no dicha renta y una determinada cuantía en función de la Comunidad Autónoma - en adelante CA- en la que resida el beneficiario lo que, claramente, ha supuesto una vulneración del principio de igualdad que debe regir nuestro ordenamiento jurídico y es por ello que se hacía necesario gestionar y articular una prestación igual para todos y cada uno de los ciudadanos para, así, no vulnerar el derecho a la igualdad que se ha materializado este mismo año con la aprobación del Real Decreto Ley 20/2020, de 29 de mayo, por el que se establece el ingreso mínimo vital -en adelante RDL 20/2020-.

Cabe recordar que la base de todos y cada uno de estos argumentos es la forma de Estado en la que el constituyente decidió organizarlo en 1978, es decir, un Estado Social y Democrático de Derecho y que, por tanto, es el propio Estado quien deberá garantizar dichas cuestiones y fomentar la protección social a través de su intervención que, como bien es sabido, se trata de una obligación legal nacida del texto constitucional.

El pasado 29 de mayo de 2020 se aprobó por Consejo de Ministros RDL 20/2020, de 29 de mayo, por el que se establece el ingreso mínimo vital ${ }^{2}$, en el que se regula la prestación

\footnotetext{
${ }^{1}$ MONEREO PÉREZ, José Luis. "La renta de "subsistencia". En especial, la renta de inserción como derecho social". En La jurisprudencia constitucional en materia laboral y social en el período 1999-2010: libro homenaje a María Emilia Casas. La Ley (España), 2015. pp. 713-755.

${ }^{2}$ Publicado en el BOE el 1 de junio de 2020 y vigencia de ese mismo día y ya con una revisión llevada a cabo solo seis días después de su aprobación -el 7 de julio de 2020-.
} 
no contributiva IMV-. Esta nueva prestación engrosa el número de prestaciones no contributivas que reconoce nuestro sistema de seguridad social. La finalidad de esta nueva prestación que ha llegado, no sin debates y polémicas, es, sin lugar a dudas, la materialización de la intervención eficaz del Estado a través de nuestro sistema de seguridad social para, así, paliar las situaciones de pobreza extrema que puedan darse en nuestro país.

En 1995, el Pacto de Toledo, ya tuvo en cuenta las tradicionales prestaciones no contributivas como parte de nuestro sistema de seguridad social y como camino inequívoco para la consecución de un sistema universal a través de una cobertura viable entre prestaciones contributivas y prestaciones no contributivas. En $2011^{3}$ se consideró necesario enmendar las prestaciones no contributivas de modo que su cobertura fuese mayor mejorando, así, las existentes y creando otras nuevas que ayudaran a mantener la esencia del Estado social pero siempre partiendo de la base de las prestaciones contributivas, creando de forma análoga una prestación no contributiva que se correspondiese con las primeras.

La introducción de esta nueva prestación en nuestro sistema de seguridad social estaba prevista ya desde el acuerdo de Gobierno ${ }^{4}$ firmado por las dos formaciones políticas que lo constituyeron el pasado 7 de enero de 2020 -PSOE y Unidas Podemos - pero la situación de crisis actual acrecentada por la aparición de la COVID-19 parece que ha contribuido a que la regulación del IMV se haya adelantado a lo inicialmente previsto.

De la propia exposición de motivos del RDL 20/2020 se desprende, de forma clara y concisa, que la aprobación de la prestación del IMV supone, sin lugar a dudas, un nuevo avance del sistema social y democrático de derecho en el que vivimos y, ante todo, una mejora indiscutible del Estado de bienestar que es, obviamente, uno de los objetivos de nuestro Gobierno, convirtiéndose en una opción para muchas personas ante la posibilidad de abandonar su situación de pobreza extrema y, así, tener acceso a las necesidades básicas para la subsistencia. No cabe olvidar que el objetivo de dicha prestación no

\footnotetext{
${ }^{3}$ En referencia y aplicación de la Recomendación $15^{a}$ del Pacto de Toledo: "Las actuales previsiones pueden alterarse significativamente en las próximas décadas. Resulta por ello del máximo interés que se utilicen instrumentos, en el Gobierno y en el Parlamento, que realicen un seguimiento y una evaluación periódica de la evolución de las magnitudes que intervienen en el equilibrio financiero le1 sistema para operar con las intervenciones que fueran requeridas en cualquier eventualidad. Esta información se trasladará a los agentes sociales. Se propone que el Congreso de los Diputados cada cinco años cree una Ponencia que estudie el presente y futuro del sistema de Seguridad Social como garantía de continuidad del mismo. Se prestará una atención específica a las consecuencias que la reciente reforma de la normativa laboral pueda tener sobre la financiación de la Seguridad Social y el sistema de prestaciones de la misma". ${ }^{4}$ Firmado el 30 de diciembre de 2019 entre Pedro Sánchez y Pablo Iglesias.
} 
contributiva es favorecer la destrucción de la pobreza asegurando -o por lo menos intentándolo- una renta mínima.

\section{El sistema de seguridad social español y las prestaciones no contributivas.}

El sistema de seguridad social español posee su justificación jurídica en el artículo $41^{5}$ de la $\mathrm{CE}$ pero es cierto que dicho mandato no era suficiente para su correcta implementación ya que regula el sistema de forma somera por lo que necesitó de regulación más específica. A lo largo de los años se fueron aprobando textos legales -entre ellos el más importante en la materia: el Real Decreto Legislativo 1/1994, de 20 de junio, por el que se aprueba el Texto Refundido de la Ley General de la Seguridad Social ${ }^{6}$-.

Atendiendo al mencionado artículo 41 de la $\mathrm{CE}$ se debe tener en cuenta que no hace referencia a un único sistema o modelo de seguridad social sino que, al contrario, obliga al Estado a promoverlo y garantizarlo a través de los mecanismos necesarios pero reconociendo la posibilidad de la existencia de su evolución lo que significa que el sistema de seguridad social español tiene un carácter público indudable -y el Estado debe ser el garante del sistema de seguridad social- pero que, no obstante, no se establecen impedimentos legales a la coexistencia con otros de carácter privado, término justificado por el Tribunal Constitucional -en adelante TC- en el Fundamento Jurídico -en adelante FJ- número $4^{\text {o }}$ de la STC 37/1994, de 10 de febrero en la que estableció que la única limitación que establece la CE es que se pongan en cuestión los rasgos estructurales de la institución de la seguridad social. Una idea clave en este sentido es, además, la establecida por el TC en el FJ $2^{\circ}$ de su STC38/1995, de 13 de febrero y en el FJ $4^{\circ}$ de la STC 77/1995, de 20 de mayo, por el que establece que la protección de todos los ciudadanos constituye un imperativo jurídico.

El gran cambio se produce con la aprobación de la Ley 26/1990, de 20 de diciembre, por la que se establecen en la seguridad social prestaciones no contributivas, que terminó de configurar el sistema de seguridad social tal y como lo había establecido el artículo 41 de la CE, es decir, introduciendo la protección a nivel contributivo y a nivel no contributivo.

\footnotetext{
${ }^{5} \mathrm{Si}$ bien es cierto que no es el único ya que otros muchos tales como, por ejemplo, el 25.2 establece las bases del trabajo remunerado, el 129.1 obliga al Estado a establecer las formas de participación de los interesados en materia de seguridad social y el 149.1.7 de la CE establece la competencia exclusiva del Estado en materia de seguridad social en cuanto a legislación básica y régimen económico. Otros preceptos legales, además, establecen obligaciones íntimamente relacionadas con el sistema de seguridad social como, por ejemplo, la protección económica de la familia regulada en el artículo 39, la protección de la salud regulada en el artículo 43 o la suficiencia económica regulada en el artículo 50, entre otros.

${ }^{6}$ Hoy derogado y sustituido por el Real Decreto Legislativo 8/2015, de 30 de octubre, por el que se aprueba el texto refundido de la Ley General de la Seguridad Social -en adelante TRLGSS.
} 
En la actualidad, la acción protectora de nuestro sistema de seguridad social parte de la idea de una protección universal ${ }^{7}$ a la que tendrán derecho todas las personas -si bien es cierto que a veces se circunscribe a ciudadanos o residentes- en igualdad de condiciones independientemente de su contribución anterior a través de las cotizaciones al sistema. La Ley 26/1990, de 20 de diciembre, supuso pues un antes y un después para nuestro sistema de seguridad social dando paso a la protección ya no únicamente a los sujetos que se encontraran -o se hubiesen encontrado- en situación del alta en nuestro sistema en base a una relación profesional sino, además, a cualquier ciudadano que resida en España ${ }^{8}$ lo que supuso, sin lugar a dudas, un avance en el camino hacia la universalidad del sistema a lo que la nueva prestación del IMV ha contribuido, del mismo modo, en gran medida.

El artículo 2 del Texto Refundido de la Ley General de Seguridad Social -en adelante TRLGSS- establece un tipo de protección referida al nivel contributivo del sistema de seguridad social que viene determinada por el cumplimiento de los requisitos de cotización establecidos legalmente y que se corresponderá con las personas que realicen o hayan realizado un trabajo por cuenta propia o ajena y que, por tanto, hayan contribuido al sostenimiento de nuestro sistema y, además, el referido al nivel no contributivo para otorgar la protección a aquellas otras personas que carezcan de recursos independientemente de que realicen o no actividades profesionales ${ }^{9}$ - lo que implica que no es necesario que haya contribuido anteriormente al sostenimiento del sistema de seguridad social. Dentro de este ámbito se puede incluir, como se verá, la nueva prestación no contributiva por IMV ${ }^{10}$.

El sistema de seguridad social español es un sistema social basado, además, en el régimen de prestaciones - en nuestro caso tanto contributivas como no contributivas- en el que las personas tiene acceso a estas a través de dos vías: la contribución al sistema a través de sus cotizaciones y el aprovechamiento de las cotizaciones de otros en los casos de necesidad - esta última base del sistema social- que es, sin lugar a dudas, la característica que cumple con el requisito de universalidad del sistema -entendiendo la universalidad

\footnotetext{
${ }^{7}$ Que incluía hasta ahora: la asistencia sanitaria, la protección familiar, los servicios sociales y, en ocasiones, el subsidio por desempleo.

${ }^{8}$ RODRÍGUEZ RAMOS, María José, GORELLI HERNÁNDEZ, Juan, VÍLCHEZ PORRAS, Marco Antonio. "Sistema de Seguridad Social" (7 edición). Madrid, Tecnos, 2005, p. 71.

${ }^{9}$ ALARCÓN CARACUEL, Manuel Ramón, "La Seguridad Social en España”, Pamplona, Aranzadi, 1999, pp. 105-108.

${ }^{10}$ No hay que olvidar que nuestro sistema de seguridad social era tradicionalmente profesionalizado pero que, desde hace tiempo, tiene un carácter puramente universal y deslaboralizador - MONEREO PÉREZ, José Luis, MOLINA NAVARRETE, Cristóbal, QUESADA SEGURA, Rosa. Manual de Seguridad Social (11 a edición). Madrid, Tecnos, 2015, p. 90- como resalta el FJ $2^{\circ}$ de la STC 3/1993, de 14 de enero que establece que El artículo 41 de la Constitución hace un diseño de la seguridad social con vocación universalista en el área de protección básica.
} 
como uno de los principios fundamentales de nuestro sistema y, por lo tanto, su pilar fundamental- cuya finalidad es acabar, en la medida de lo posible, con las situaciones de necesidad que puedan surgirle a las personas con el transcurso de los años. Nuestro sistema es, pues, un sistema público y colectivo que persigue la implantación absoluta establecida por el mandato Constitucional del Estado de Bienestar puro y completo ${ }^{11}$ mandato que todavía a día de hoy, casi cuatro décadas después de su establecimiento, no se ha alcanzado en su totalidad-.

Dicho esto, no podemos olvidar que nuestro sistema de seguridad social se basa en siete principios básicos ${ }^{12}$ de entre los cabe destacar en estas líneas el principio de solidaridad. Dicho principio es esencial para la consecución de la garantía del principio de universalidad del sistema de seguridad social y, por ende, se hallan íntimamente relacionados entre sí. La base principal es que cualquier miembro de la población está obligado, en la medida de sus posibilidades, a contribuir económicamente al mantenimiento del sistema lo que se materializa a través de las cotizaciones de empleadores y trabajadores - por cuenta propia o ajena- y que supone que toda la comunidad pueda percibir las prestaciones que le puedan corresponder o aquellas necesarias para garantizar una vida digna -como es el caso de la prestación del IMV-.

El principio de solidaridad es el que, sin duda, sustenta el sistema de seguridad social y permite la configuración efectiva del Estado Social - la configuración del mismo viene consagrado en el artículo 1.1 de la $\mathrm{CE}-$. Se puede señalar que el principio de solidaridad es la materialización de la efectividad del sistema público de seguridad social consagrado en la CE y que, por ende, se le otorga el carácter vinculante y obligatorio que lo caracteriza. Un sistema de seguridad social basado únicamente en el sistema de cotizaciones no supondría el disfrute de un sistema solidario que se traduciría en el olvido de los más necesitados.

La solidaridad como principio debe entenderse desde varias vertientes ${ }^{13}$ : la solidaridad entre generaciones ${ }^{14}$, la solidaridad entre diferentes sectores de producción ${ }^{15}$ y la solidaridad entre rentas ${ }^{16}$.

\footnotetext{
${ }^{11}$ GARCÍA DÍAZ, Miguel Ángel. "La seguridad social en España: Situación actual y algunas reflexiones sobre su futuro". Cuadernos De Relaciones Laborales, (8), 1996, pp. 85-105.

${ }^{12}$ Publificación institucional, Universalidad, Unidad, Solidaridad, Igualdad, Obligatoriedad y Generalidad y Principio de Suficiencia.

${ }^{13}$ GARCÍA DÍAZ, Miguel Ángel. "La seguridad social en España: Situación actual y algunas reflexiones sobre su futuro". Op. Cit., pp. 85-105.

${ }^{14}$ Con las cotizaciones y aportaciones actuales se deben financiar las pensiones de la generación anterior.

${ }^{15}$ Cuando un sector resulte deficitario para el sistema el que posea superávit será quien sustente al primero.

${ }^{16}$ Estableciendo mayores bases de cotización a las rentas más altas para, así, garantizar las pensiones mínimas a aquellas personas con menores ingresos.
} 
Hay que subrayar que el catálogo de derechos que existe en nuestro ordenamiento jurídico no es cerrado y que, por tanto, avanza en función de las necesidades de cada momento en concreto y, por tanto, el legislador debe tomar en consideración las necesidades y demandas que aparezcan en función de la situación económica y social de cada momento histórico. En el caso que se aborda es de resaltar que se trata de proporcionar cobertura suficiente a ciertos colectivos susceptibles de vulnerabilidad que pretenden protegerse a través de esta nueva prestación de nuestro sistema de seguridad social. El Estado debe garantizar la creación de mecanismos de este tipo como garante de nuestro sistema y, por tanto, proteger la dignidad de las personas lo que conlleva, sin lugar a dudas, la protección de nuestro tipo de Estado consagrado por la Constitución. Ello supone una clara intervención del Estado, la fomenta y la consolida como obligación inequívoca de este y, por ende, del resto de poderes públicos vinculados al mismo ${ }^{17}$.

\section{El carácter de prestación no contributiva del Ingreso Mínimo Vital}

La prestación por IMV se trata de una prestación no contributiva; partiendo de esa base cabe recordar que dichas prestaciones deben estar garantizadas por el Estado a todas aquellas personas que tenga derecho a cualquiera de las prestaciones protegidas en las que no exista requisito de cotización o contribución previa; como ya indiqué, se trata de un nivel asistencial que se materializa con la existencia de prestaciones no contributivas ${ }^{18}$ y cuya finalidad es la universalización del sistema de seguridad social ${ }^{19}$. El propósito no es otro más que garantizar la protección eficaz, eficiente y suficiente a cualquier persona que se encuentre en situación de necesidad por el mero hecho de serlo si bien es cierto que, hoy en día, todavía tiene carencias destacables pero, sin lugar a dudas, la inclusión en el catálogo de prestaciones de la prestación no contributiva por IMV ha ampliado el ámbito de protección existente hasta la actualidad; no obstante, todavía es necesario que los individuos reúnan una serie de requisitos legales que les permitan el acceso a dichas prestaciones $^{20}$.

\footnotetext{
${ }^{17}$ MONEREO PÉREZ, José Luis. "Nuevos derechos para nuevas situaciones de necesidad y de emergencia social: el ingreso mínimo vital". Estudios financieros. Revista de trabajo y seguridad social: Comentarios, casos prácticos: recursos humanos, $\mathrm{n}^{\circ} 448,2020$, pp. 27-68.

${ }^{18}$ Que, tradicionalmente, eran la asistencia sanitaria y farmacéutica, las pensiones no contributivas de jubilación o invalidez, prestaciones por razón de necesidad, pensión asistencial de necesidad, prestaciones familiares económicas, subsidios por desempleo, subsidio no contributivo por maternidad y servicios sociales - ALARCÓN CARACUEL, Manuel Ramón. La Seguridad Social en España. Pamplona, Aranzadi, 1999, pp. 105-108-.

${ }^{19}$ ALONSO OLEA, Manuel., TORTUERO PLAZA, Jose Luis. Instituciones de Seguridad Social (18 ${ }^{\mathrm{a}}$ rev edición). Madrid: Civitas, 2002, p. 50

${ }^{20}$ MONEREO PÉREZ, José Luís, Cristobal MOLINA NAVARRETE, Rosa QUESADA SEGURA. Manual de Seguridad Social Op. Cit., p. 90.
} 
La exposición de motivos RDL 20/2020, establece de manera clara que su finalidad es la misma que la de cualquier otra prestación no contributiva, es decir, suplir un estado de necesidad cierto y demostrado y asegurar una renta mínima a aquellos hogares en situación de vulnerabilidad para, así, poder reducir la pobreza extrema presente en nuestro país basando dicha prestación en el estado de necesidad de forma preferente frente a otros criterios y entendiéndolo como un estado de carencia real y efectiva de recursos económicos que, de no subsanarse, dejarían a las personas por debajo del umbral de subsistencia. Cierto es que se ha configurado la prestación por IMV como una prestación no contributiva, pero podría decirse que, quizá, no es la categorización más exacta ya que, tradicionalmente, se constituyen como prestaciones alternativas y excluyentes de las contributivas -al poseer otra prestación paralela en el elenco de prestaciones contributivas $^{21}-\mathrm{y}$ esto no sucede en el caso del IMV.

La norma determina la configuración de la prestación por el IMV como una prestación de carácter económico cuya finalidad es la garantía de la obtención de ingresos mínimos suficientes a aquellos sujetos $-y$ sus beneficiarios- que se encuentren en situación de especial vulnerabilidad debida al contexto económico que rigen su entorno que pretende mejorar, como ya he indicado con anterioridad, el acceso a la inclusión tanto social como laboral de los sujetos que sufren situaciones de necesidad.

Es por todo lo anterior que, el legislador, ha incluido esta prestación en el catálogo de prestaciones de nuestro sistema de seguridad social y, en concreto, a los recogidos en el artículo 42.1.c) del TRLGSS. El IMV posee cuatro notas definitorias fundamentales: garantizar un nivel mínimo de renta tanto del beneficiario como de sus dependientes, establecer el nivel de protección en función de las características de la unidad de convivencia -que se definirá en el apartado posterior-, la concesión de la misma con carácter indefinido ${ }^{22}$ y, además, su carácter personal o grupal e intransferible.

La creación y aprobación de la prestación por IMV es, sin lugar a dudas, muestra de la evolución del sistema que atiende a esa característica mencionada ya a lo largo de estas líneas y que refiere al cambio continuo al que debe estar supeditado nuestro ordenamiento jurídico en general y el referente a seguridad social en particular. Al tratarse de una prestación de carácter no contributivo resultan clarificadoras las SSTC 37/1994 y 65/1987 que establecen que la seguridad social es -y debe ser- una función del Estado y, por ende, el estándar multinivel de garantía del derecho a la seguridad social ${ }^{23}$. El carácter no

\footnotetext{
${ }^{21}$ Excepto en el caso del complemento a mínimos.

${ }^{22} \mathrm{Si}$ bien es cierto que es susceptible de revisión lo que supone que el beneficiario dejará de ser susceptible de percibir la prestación.

${ }^{23}$ MONEREO PÉRE, José Luis Z. "Nuevos derechos para nuevas situaciones de necesidad y de emergencia social: el ingreso mínimo vital”. Op. Cit., pp. 27-68.
} 
contributivo de la prestación supone que la protección sea igualitaria independientemente de la $\mathrm{CA}$ en la que resida el beneficiario y, por tanto, para todas las personas.

Lo coherente en este sentido era que la nueva prestación pasara a integrarse en el TRLGSS $^{24}$ si bien es cierto que sorprende que se trate de una prestación de carácter no contributivo que se constituye en el citado texto legal, pero lo hace ex Título VI encargado de regular este tipo de prestaciones-.

Recapitulando, se puede decir que el IMV se regula ex novo, sin prestación paralela en la rama contributiva y sin entenderse complementaria a ninguna de las prestaciones de dicho catálogo; por otro lado, no podríamos considerar el IMV como asistencia social interna de nuestro sistema de seguridad social ya que su nivel de protección pretende complementar ambas vertientes del sistema.

\section{El Ingreso Mínimo Vital, en síntesis}

El TC en STC 206/1997, de 27 de noviembre ${ }^{25}$ indicó de forma clarificadora que el artículo 41 de la CE no impide cambios en el sistema de seguridad social que implique modificaciones que propicien la mejora de este. Se debe partir de la base fijada por el mencionado artículo constitucional y explorar todas las cuestiones o controversias encaminadas a establecer ese elenco de prestaciones suficientes que debe caracterizar nuestro sistema político y, por tanto, el de seguridad social.

Como se verá en epígrafes posteriores, el IMV se ha configurado como un mínimo, es decir, no se establece de forma universal para todos y cada uno de los ciudadanos, sino que está supeditado a ciertas circunstancias personales y económicas que van a determinar el derecho de acceso a la prestación.

El RDL 20/2020 se concreta en una norma compuesta por treinta y siete artículos, cinco disposiciones adicionales, siete disposiciones transitorias, una disposición derogatoria, onces disposiciones finales y dos anexos; todo ello para concretar una prestación que se ha hecho de esperar a lo largo de los últimos años. El RDL regula todas y cada una de las cuestiones que componen la nueva prestación no contributiva de nuestro sistema de seguridad social que supone la regulación de los sujetos protegidos -y sus beneficiarios-, la acción protectora, su procedimiento de tramitación y resolución, financiación y, por supuesto, obligaciones, infracciones y sanciones.

\footnotetext{
${ }^{24}$ Aprobado por RD legislativo 8/2015, de 30 de octubre.

${ }^{25}$ BOE núm. 312, de 30 de diciembre de 1997.
} 
La propia exposición de motivos del RDL 20/2020 basa la justificación de su existencia en una cuestión fundamental y es que España se encuentra entre los países pertenecientes a la UE que más desigualdad a nivel económico demuestra entre sus ciudadanos y, por ende, de pobreza extrema lo que es muestra del débil efecto redistributivo del conjunto de la intervención del Estado en España en comparación con la mayoría de los países de nuestro entorno lo que supone un menor desarrollo y financiación de partidas con claro impacto redistributivo ${ }^{26}$.

La prestación por IMV otorga, sin lugar a dudas, una solución homogénea a todos y cada uno de los ciudadanos del país teniendo en cuenta las mismas características evaluadoras de acceso a la prestación. Se ha convertido en una prestación fundamental y necesaria dentro de nuestro ordenamiento jurídico que, además, incluye políticas incardinadas a la inclusión social del beneficiario de la misma y cuya pretensión es la inclusión social y económica del individuo ${ }^{27}$.

No existe mejor manera de expresar la pretensión de la nueva prestación que aclarar su finalidad a tenor de lo establecido en el artículo 1 de la propia norma que establece que “...tiene por objeto la creación y regulación del ingreso mínimo vital como prestación dirigida a prevenir el riesgo de pobreza y exclusión social de las personas que vivan solas o integradas en una unidad de convivencia, cuando se encuentren en una situación de vulnerabilidad por carecer de recursos económicos suficientes para la cobertura de sus necesidades básicas".

\subsection{Conceptos}

Con la aprobación de esta nueva prestación no contributiva hay una serie de conceptos que debían regularse y definirse; así pues, aparecen algunos tales como: IMV, Unidad de convivencia o Vulnerabilidad Económica.

\section{a. Ingreso Mínimo Vital}

El $I M V$ es una nueva prestación no contributiva establecida dentro del catálogo de prestaciones de nuestro sistema de seguridad social cuya finalidad es prevenir el riesgo de pobreza y exclusión social.

\footnotetext{
${ }^{26}$ MONEREO PÉREZ, José Luis. "Nuevos derechos para nuevas situaciones de necesidad y de emergencia social: el ingreso mínimo vital”. Op. Cit., pp. 27-68.

${ }^{27}$ En otras cuestiones se pretende fomentar la participación de los beneficiarios en el mercado laboral con incentivos que cubren dicha finalidad como, por ejemplo, rebajar la cuantía del salario cuando el beneficiario encuentre trabajo para que no deje de percibir la prestación o la obligatoriedad de inscribirse como demandante de empleo.
} 
La propia exposición de motivos del RDL 20/2020 establece que el IMV está configurado como el derecho subjetivo a la percepción de una prestación de naturaleza económica cuyo objetivo es garantizar un nivel mínimo de renta para aquellas personas que se encuentren en situación de vulnerabilidad económica -en los términos que la norma establezca- de modo que se debe entender su finalidad como la destinada a suplementar la renta de cada solicitante y el umbral fijado legalmente para cada tipo de beneficiario o unidad de convivencia.

\section{b. Unidad de Convivencia}

Con carácter general, se considera unidad de convivencia la constituida por todas y cada una de las personas que residan en un mismo domicilio y que se encuentren unidas entre sí por vínculo matrimonial, pareja de hecho o incluso por vínculo de hasta el segundo grado de consanguineidad o afinidad así como por adopción, guarda o acogimiento familiar; ahora bien, la norma es mucho más extensa y realiza una consideración amplia de la misma incluyendo, además, la constituida por una persona víctima de violencia de género o que haya iniciado los trámites de separación o divorcio -independientemente de que sea o no víctima de violencia de género- que haya debido abandonar su domicilio familiar habitual siempre y cuando esté acompañada de sus hijos -o menores a su cargo o familiares de hasta el segundo grado de consanguineidad o afinidad-. Otra de las situaciones reconocidas como unidad de convivencia es aquella que hace referencia a la constituida por dos o más personas con un rango de edad de entre veintitrés y sesenta y cinco años que, independientemente de la relación que ostenten entre ellos, convivan en el mismo domicilio cumpliendo con las exigencias que la norma establezca en cada caso $^{28}$.

\section{c. Vulnerabilidad Económica}

La vulnerabilidad económica vendrá determinada en función de la capacidad económica del solicitante del IMV como beneficiaria individual o según la correspondiente a la unidad de convivencia en su conjunto teniendo en cuenta la totalidad de los recursos a los que tienen acceso cada uno de los miembros ${ }^{29}$ de modo que, cuando sea inferior en, al

\footnotetext{
${ }^{28}$ En este caso se considerarán tantas cuantas unidades de convivencia se consideren existentes en función de los supuestos anteriores; así pues, solo se considerará unidad de convivencia cuando exista relación entre los miembros de la misma y será posible la convivencia en un mismo domicilio de varias unidades de convivencia.

${ }^{29}$ Los inmuebles -quedando excluida la vivienda habitual-, las cuentas bancarias y depósitos, los activos financieros -valores, seguros, rentas y participaciones en instituciones de inversión colectiva- y las participaciones en planes o fondos de pensiones -u otros similares-.
} 
menos diez euros, a la cuantía mensual de la renta garantizada con esta prestación ${ }^{30}$ según modalidad y número de miembros que compongan la unidad de convivencia- de modo que no existirá vulnerabilidad económica en caso de que el beneficiario individual del IMV sea titular de un patrimonio cuyo valor sea equivalente o superior a tres veces las cuantía de la renta garantizada por el IMV para un beneficiario individual mientras que para el caso de las unidades de convivencia habrá que aplicar una escala de incrementos regulada legalmente ${ }^{31}$.

\subsection{Requisitos y Características}

El IMV está pensado con la finalidad de proteger a personas de entre veintitrés y sesenta y cinco años ${ }^{32}$ que puedan acreditar que se encuentran en situación de vulnerabilidad económica debido a la carencia de rentas, ingresos o patrimonio con los que sustentarse y garantizar el disfrute de las necesidades básicas ${ }^{33}$.

Dichas personas deben cumplir una serie de requisitos legalmente establecidos como, por ejemplo, tener residencia legal y efectiva en España de forma continuada e ininterrumpida con una duración mínima de un año - dicho año debe ser inmediatamente anterior a la fecha de presentación de la solicitud de la prestación por IMV $^{34}$ - o el estudio del patrimonio neto del solicitante ${ }^{35} \mathrm{y}$, por supuesto, encontrarse en situación de vulnerabilidad económica y haber vivido de forma independiente ${ }^{36}$, por lo menos, con tres años de anterioridad a la solicitud de la prestación por IMV.

\footnotetext{
${ }^{30}$ Sin tener en cuenta los salarios sociales, las rentas mínimas de inserción o las ayudas análogas otorgadas por las Comunidades Autónomas.

${ }^{31}$ En el caso de un único solicitante, su patrimonio no podrá ser superior a $600 €$ mientras que en hogares de cuatro o más personas no podrán superar un patrimonio de $43.000 €$-hay que recordar que queda excluida de dicho cómputo la vivienda habitual-.

${ }^{32} \mathrm{Si}$ bien es cierto que podrán, además, acceder todas las personas mayores de edad o menores emancipados siempre y cuando tengan hijos o menores en régimen de guarda.

${ }^{33}$ Quedando excluidas aquellas personas que sean administradores de derecho de una sociedad mercantil el solicitante en caso de que lo haga de forma individual o cualquiera de los miembros de la unidad de convivencia en caso de que se opte por dicha modalidad-independientemente de la valoración que se realice su patrimonio.

${ }^{34}$ Tanto este requisito como el de la edad no serán de obligado cumplimiento para aquellas personas que acrediten ser víctimas de trata de seres humanos y de explotación sexual y las mujeres que hayan sido víctimas de violencia de género.

${ }^{35}$ Que hace referencia a la totalidad de su patrimonio excluyendo de este tanto las deudas que tuviese suscritas como su vivienda habitual.

${ }^{36}$ Se entenderá que una persona ha vivido de forma independiente si ha permanecido en situación de alta en cualquiera de los regímenes que integran el sistema de la Seguridad Social durante al menos 1 año, continuado o no, y siempre que acredite que su domicilio ha sido distinto al de sus progenitores, tutores o acogedores durante 3 años anteriores a la solicitud.
} 
Si los beneficiarios forman parte de una unidad de convivencia, además, se exigirá un plazo de constitución de la misma de al menos un año anterior a la presentación de la solicitud de la prestación.

Las personas que deseen acceder a la prestación por IMV deberán, además, haber solicitado y agotado todas y cada una de las prestaciones de nuestro sistema de seguridad social a las que pudiesen tener derecho de acceso para acogerse a esta nueva prestación y, además, figurar inscritos como demandantes de empleo ${ }^{37}$.

\subsection{Plazo de presentación}

En lo que se refiere al plazo de presentación de solicitudes para la obtención del IMV hay que destacar que comenzó el 15 de junio de 2020 si bien es cierto que todas aquellas solicitudes aceptadas tendrán carácter retroactivo con fecha 1 de junio de 2020 -siempre y cuando se presenten entre el 15 de junio y el 15 de septiembre de 2020. En caso contrario se entenderá concedida desde el primer día del mes siguiente al de presentación de la solicitud-.

\subsection{Duración}

Lo primero que hay que destacar es que se trata de una prestación no contributiva y que, además, tiene carácter permanente, es decir, una vez reconocido el derecho y siempre y cuando no existan cambios en las condiciones y circunstancias que generaron el derecho a la percepción el beneficiario seguirá teniendo derecho a la percepción de la misma con carácter permanente.

Para determinar dicha circunstancia será la propia seguridad social quien realice los perceptivos controles regulares que justifiquen que la situación del beneficiario no ha variado en relación al momento de la concesión de la misma. En este sentido hay que destacar que en caso de haber algún cambio sustancial es el propio perceptor el obligado a comunicarlo en un plazo no superior a treinta días naturales desde que este se hubiese producido.

\footnotetext{
${ }^{37}$ Cuando las personas que deseen acceder a la prestación IMV no sean beneficiarios de prestaciones o subsidios de desempleo y a los efectos únicos del cómputo de la renta se podrán presentar solicitudes hasta el 31 de diciembre de 2020 siempre que la vulnerabilidad se haya producido en el año en curso -a mi parecer otra medida encaminada a subsanar ciertas situaciones acaecidas a raíz del COVID-19-. En este supuesto se tendrá en cuenta la parte proporcional de los ingresos de la unidad familiar de convivencia en 2020 siempre que en 2019 la renta no supere la mitad de los límites del patrimonio neto establecidos de forma general.
} 


\subsection{Solicitud}

El sistema de solicitud del IMV es relativamente sencillo y, por ello, se puede realizar a través de la sede electrónica de la seguridad social ${ }^{38}$ o a través del envío de la documentación por correo ordinario.

Para garantizar la viabilidad de concesión dicha solicitud debe ir firmada por todas aquellas personas que compongan la unidad de convivencia siempre y cuando sean mayores de edad y no se encuentren incapacitados legalmente.

\subsection{Reconocimiento y control}

El sistema de reconocimiento y control de la prestación recae íntegramente en el Instituto Nacional de la Seguridad Social -en adelante INSS- que dispone de un plazo de tres meses desde la recepción de la solicitud para resolver el reconocimiento o no del IMV.

\subsection{Obligaciones, Infracciones y Sanciones}

El propio RDL 20/2020 establece un régimen especial de infracciones y sanciones e instituye que tanto los titulares de la prestación no contributiva por IMV como los beneficiarios de la misma que hayan cometido infracción serán responsables de las mismas.

Dichas infracciones ${ }^{39}$ se tipifican en leves, graves y muy graves y podrán suponer la obligación a la devolución de la prestación indebidamente percibida, la extinción del derecho o la imposibilidad de acceso a la citada prestación en momentos posteriores.

\subsection{Cuantía}

Se han establecido diferentes baremos para establecer la cuantía de la prestación diferenciada en 14 categorías distintas en función de la situación familiar. Para el establecimiento de las mismas se ha realizado un trabajo conjunto entre el Instituto Nacional de Seguridad Social -en adelante INSS- y la Agencia Tributaria -en adelante AT- que sufren un incremento del $0.3 \%$ por cada adulto o menor e instaura un incremento del $0.22 \%$ en el caso de hogares monoparentales ${ }^{40}$.

\footnotetext{
${ }^{38}$ www.seg-social.es

${ }^{39}$ Pueden responder al falseamiento de documentación o situación personal o de cualquier miembro de la unidad de convivencia, ocultación fraudulenta de cambios en la situación del titular beneficiario o de cualquiera de los miembros de la unidad de convivencia o cualquier otra actuación u omisión que otorgue derecho indebido a la percepción de la prestación por IMV.

${ }^{40}$ Denominadas escalas de incremento.
} 
En lo que se refiere a las cuantías y en función de lo establecido en el párrafo anterior se establece, por ejemplo, que un beneficiario único tendría derecho a la percepción de $5.640 €$ anuales en concepto de IMV -lo que supone un total de $470 €$ mensuales repartidos en doce mensualidades- y una unidad de convivencia formada por un adulto y un niño percibiría en concepto de IMV un total de $8.418 €$ anuales, es decir, lo $470 €$ mensuales más el incremento de $140 €$ mensuales por miembro adicional. El pago de la misma se realizará mediante transferencia bancaria al beneficiario de la prestación con periodicidad mensual.

De sobra es conocida la situación social actual derivada de la pandemia causada por la COVID-19 por lo que el RDL 20/2020 no ha querido dejar de prestar especial atención a dicha situación de modo que se ha previsto un procedimiento excepcional que asegure que el IMV alcance de manera urgente a los más vulnerables por lo que para establecer la percepción de la cuantía se tendrán en cuenta los ingresos percibidos en 2020 -en lugar de aplicar el baremo del año anterior reconocido en la norma con carácter general-.

En el caso de que haya cambios en las unidades de convivencia, causas personales, económicas o patrimoniales tanto del beneficiario como de alguno de los miembros de la unidad de convivencia se realizará la pertinente revisión por parte del INSS lo que podrá suponer variación en el importe a percibir por el beneficiario que se podrá traducir tanto en el aumento como en la disminución de la cuantía de la prestación ${ }^{41}$.

El Gobierno será quien actualizará los valores, escalas y porcentajes establecidos en el RDL 20/2020 en función de las circunstancias que se vayan produciendo en nuestro país para adaptarlos a los cambios sociales y económicos y, por supuesto, a las situaciones de vulnerabilidad de los individuos con el único fin de que la prestación de IMV mantenga su acción protectora de forma íntegra y no pierda su razón de ser.

\section{El Ingreso Mínimo Vital: Compatibilidades e incompatibilidades entre prestaciones}

La primera cuestión que se hace necesario poner de manifiesto es que el IMV no se ha creado con la idea de hacer desaparecer el resto de prestaciones de nuestro sistema de seguridad social sino como un complemento a las mismas para conseguir eliminar, en la medida de los posible, la vulnerabilidad económica que sufren un gran número de personas en nuestro país y que es incompatible con nuestro sistema de Estado y, por

\footnotetext{
${ }^{41}$ De oficio se realizarán revisiones cada año natural que supondrán modificaciones a partir del 1 de enero del año siguiente.
} 
supuesto, con nuestro sistema de seguridad social dados los principios en los que ambos se sustentan.

Nuestro sistema de seguridad social se encarga de regular, establecer y repartir los ingresos referentes a las dos ramas que lo componen: la contributiva y la no contributiva -sustento de un Estado Social como el nuestro-. En este sentido se debe recordar que la vertiente contributiva de las prestaciones de la seguridad social es el principal modo de reparto de ingresos que, además, sustenta el sistema de cobertura previsto por nuestro sistema de seguridad social que se nutre de las cotizaciones de las personas trabajadoras en activo para hacer frente a las prestaciones de individuos que, por una u otra razón, no lo están -bien de forma permanente o bien de forma transitoria-.

El RDL 20/2020 hace referencia al IMV como una prestación englobada dentro del catálogo de prestaciones no contributivas de nuestro sistema de seguridad social pero hay que matizar que, tradicionalmente, este tipo de prestaciones eran la alternativa para aquellas personas que no tenían derecho a la percepción de una pensión contributiva ${ }^{42}$. Dicho RDL está pensado, sin lugar a dudas, para garantizar la protección de aquellas personas que se encuentran en un claro estado de necesidad y su finalidad principal es avalar una prestación que resulte suficiente para responder a las necesidades mínimas de subsistencia, ahora bien, se trata de una prestación paralela al elenco de las ya existentes y que, además, no cuenta con vertiente contributiva de la misma.

A priori, la prestación por IMV es compatible con todas las prestaciones siempre y cuando no se supere el límite máximo de ingresos a percibir -excepto con las de jubilación ${ }^{43}$ pero es incompatible, sin embargo, la percepción de la prestación de IMV con la asignación económica por hijo o menor acogido a cargo -independientemente de que exista o no discapacidad inferior al 33\%- existiendo el derecho de opción entre ambas.

En el caso de la asignación económica por hijo o menor acogido a cargo hay que destacar que no se configuraba acorde a la realidad y a las necesidades de muchos de sus

\footnotetext{
${ }^{42}$ Establecido de forma clara y concisa en la Ley 26/1990, de 20 de diciembre, por la que se establecen en la seguridad social prestaciones no contributivas -hoy en día derogada- que se encargaba de regular de forma exhaustiva el artículo 41 de la Constitución Española que regula, con carácter general, nuestro sistema de seguridad social y que establece como base que tenga cobertura para todos los ciudadanos.

${ }^{43}$ La razón es sencilla en el caso de beneficiario único: la pensión mínima de jubilación establecida para los menores de 65 años es de $604.40 €$ mensuales, la de viudedad de $517.80 €$ mensuales por lo que en todos los supuestos se superaría el importe establecido por el IMV -462€-; cuestión diferente sería si se tratase de una unidad de convivencia en cuyo caso habría que realizar el cómputo correspondiente y se percibiría la diferencia a percibir en función del número de personas que formen la misma y la cantidad de ingresos percibidos en conjunto.
} 
beneficiarios $^{44}$. El RDL 20/2020 establece la incompatibilidad ${ }^{45}$ entre la prestación por IMV y la asignación económica por hijo o menor acogido a cargo por lo que se determina la eliminación de la misma para, así, considerarla incluida en la nueva prestación ${ }^{46}$ si bien es cierto que dicho cambio no afecta a aquellos solicitantes que no tengan derecho a la precepción de la prestación por IMV que continuarán percibiéndola hasta que dejen de concurrir los requisitos y proceda su extinción ${ }^{47}$.

Era tradicional que las prestaciones no contributivas no fuesen compatibles con otro tipo de ingresos como, por ejemplo, el cobro de una nómina por la realización de un trabajo por cuenta ajena o los ingresos por la realización de trabajos por cuenta propia pero una de las novedades introducidas para esta prestación en concreto es que el IMV es compatible con ambos supuestos siempre y cuando no se alcance la renta susceptible de garantía anual; es decir, el IMV es compatible con todos los ingresos provenientes del empleo por cuenta ajena o propia siempre y cuando no supere el límite establecido legalmente y así lo pone de manifiesto el artículo 33.1.g) en relación con el artículo 8.4 del RDL 20/2020.

El IMV, además, es compatible con otro tipo de ayudas que puedan provenir de Gobiernos Autonómicos y Locales y que tengan por objeto la misma protección y así lo establecen los artículos 7.1.c, 8.2 y 18.1.e.1 del RDL 20/2020, si bien es cierto que cada CA tendrá autonomía para determinar la concesión o no de la que a ella corresponde.

Como ya enuncié el IMV es compatible con otra serie de pensiones y prestaciones de las establecidos en el elenco de nuestro sistema de seguridad social si el beneficiario no supera los límites establecidos para la compatibilidad de las mismas y que, además, se hayan solicitado los complementos a las mismas -a tenor de lo establecido en el artículo 59 del TRLGSS- y así lo establece el artículo 7.1.c en relación con los artículo 8 y 18.1.d del Real-Decreto-Ley 20/2020, de 29 de mayo, por el que se establece el ingreso mínimo vital.

En referencia a las incompatibilidades es de destacar que el propio RDL 20/2020 en su artículo 16 establece que existe incompatibilidad entre la percepción del IMV y la

\footnotetext{
${ }^{44}$ Hay que apuntar que para 2020 se establecía una cuantía de $341 €$ anuales.

${ }^{45}$ Así lo establece el artículo 16 del RDL 20/2020 con la excepción de los hijos menores de 18 años con discapacidad superior al $33 \%$ o mayores de 18 con discapacidad superior al $65 \%$.

${ }^{46}$ Así lo establece la DT 7 $7^{\text {a }}$ del RDL 20/2020 que elimina la posibilidad de solicitar dicha asignación siempre y cuando se trate de menores sin discapacidad o siempre que esta no supere el $33 \%$.

${ }^{47}$ ÁLVAREZ CORTÉS, Juan Carlos. "Una nueva prestación no contributiva de la Seguridad Social: el ingreso mínimo vital. Análisis del Real Decreto-Ley 20/2020, de 29 de mayo, por el que se establece el ingreso mínimo vital". Revista General de Derecho del Trabajo y de la Seguridad Social, nº 56, 2020, p. 285 y ss.
} 
asignación económica por hijo o menor acogido a cargo, sin discapacidad o siendo esta menor al $33 \%$ pero mantiene el derecho de opción entre ambas ${ }^{48}$. Dado el carácter de la norma se establecen una serie de criterios en relación a la asignación económica por hijo o menor acogido a cargo de modo que, por un lado, la Disposición Transitoria $1^{\text {a }}$-en adelante DT- establece que se reconocerá el derecho a la percepción del IMV a aquellos que en la actualidad perciban la asignación económica por hijo o menor a cargo que reúnan los requisitos necesarios para ser beneficiarios de esta nueva prestación no contributiva siempre y cuando el ingreso sea igual o superior al que vienen percibiendo en la actualidad -nunca en el caso de que sea inferior-y, por otro, la DT $7^{\text {a }}$ que establece la eliminación de la solicitud de asignación económica por hijo o menor acogido a cargo -sin discapacidad o con discapacidad inferior al 33\%- desde el momento en el que se apruebe el RDL 20/2020, es decir, a partir del 1 de junio de 2020, si bien es cierto que aquellos que no puedan optar por el IMV podrán seguir accediendo a la misma.

En el caso de la pensión de orfandad o en favor de familiares hay que tener en cuenta que la cuantía máxima está fijada en $208.9 €$ mensuales lo que, a priori, daría acceso a la prestación por IMV a una única persona pero hay que recordar que el derecho a la prestación por orfandad finaliza al cumplir el beneficiario 21 años ${ }^{49}$ y la prestación por IMV no puede solicitarse hasta los 23 por lo que su incompatibilidad viene generada no por el rango de ingresos sino por el rango de edad establecido para cada una de las citadas prestaciones; sin embargo, dicha prestación sí computará a los efectos de la solicitud de la prestación por IMV cuando de una unidad de convivencia se trate.

En el caso de las personas beneficiarias de la pensión de jubilación debemos recordar que la norma establece que tendrán derecho a la percepción de la prestación por IMV aquellas personas que se encuentren en el rango de edad de entre veintitrés y sesenta y cinco años por lo que únicamente serán objeto de solicitud aquellas personas que cumplan con dicho requisito $^{50}$ y que, además, perciban una prestación por jubilación no contributiva y ello es porque la cuantía para esta prestación está establecida en 395,60€ mensuales divididos en catorce pagas. En este caso la prestación del IMV cubriría la diferencia de ingresos entre una y otra prestación -en este caso la diferencia estaría establecida en $98,90 €$ mensuales-. En este caso concreto hay que hacer especial mención a la cuestión de las mujeres ya que, como bien es sabido, en su gran mayoría son las que cobran las pensiones

\footnotetext{
${ }^{48}$ Si bien es cierto que de manera transitoria habrá que estar a lo establecido en la DT $1^{\mathrm{a}}$ y la DT $7^{\mathrm{a}}$.

${ }^{49}$ Salvo en excepciones tasadas legalmente y que amplían el rango de percepción hasta los 25 años o hasta que la persona sea independiente económicamente por tener un trabajo asalariado lo que supone la eliminación de la pensión independientemente de la edad.

${ }^{50}$ No afectando a los jubilados con derecho a la prestación contributiva mínima por superar la renta mínima vital recogida en el Real Decreto-Ley 20/2020, de 29 de mayo, por el que se establece el ingreso mínimo vital.
} 
más bajas de todo nuestro sistema -una razón fundamental es que son las que tradicionalmente han realizado trabajos con jornadas inferiores a la máxima legal establecida y, en la mayoría de los casos, suponía trabajos a media jornada o que, además, no han podido cotizar durante el tiempo mínimo exigible legalmente-.

Para determinar, de manera general, las características generales de compatibilidad e incompatibilidad entre prestaciones tanto contributivas como no contributivas de nuestro sistema de seguridad social y el IMV se debe tener en cuenta que el solicitante debe, en primer lugar, haber agotado las vías de solicitud de cualquiera de las prestaciones que pudiesen corresponderle como, por ejemplo, la prestación por desempleo y, en su caso el perceptivo subsidio y, después, la Renta Activa de Inserción ${ }^{51}$-siempre y cuando pueda tener acceso a la misma- y el subsidio extraordinario por desempleo regulado en la Disposición Adicional 27 $7^{\mathrm{a}}$-en adelante DA-del TRLGSS ${ }^{52}$ y solicitar incorporarse, en su caso, al Programa de Activación para el Empleo ${ }^{53}$; si no ha sido así, no podrá solicitar el IMV.

Por otro lado, existe compatibilidad, además, con cualquier ayuda que las personas puedan solicitar y percibir por la asistencia a acciones formativas para el empleo -suelen percibirse en concepto de becas para transporte, dietas, guarderías, comedores infantiles e incluso indemnizaciones por asistencia a dichas acciones de formación-.

\section{El carácter universal del Ingreso Mínimo Vital}

La idea de la articulación de una norma que garantizara, con carácter general, los ingresos insuficientes de ciertos colectivos desprotegidos en nuestro país lleva siendo una idea desde hace más de una década si bien la materialización de la misma se ha dilatado en el

\footnotetext{
${ }^{51}$ Regulada por el Real Decreto 1369/2006, de 24 de noviembre, por el que se regula el programa de renta activa de inserción para desempleados con especiales necesidades económicas y dificultad para encontrar empleo y que afecta a mayores de 45 años inscritos ininterrumpidamente como desempleados durante un periodo de doce o más meses que se comprometan a su real inserción social y que, además, carezcan de rentas de cualquier naturaleza superiores en cómputo mensual al 75\% del SMI excluida la parte proporcional de las pagas extraordinarias pero para lo que, por el contrario, sí computarán las rentas que puedan percibir de los Gobiernos Autonómicos.

${ }^{52}$ Parados de larga duración que carezcan de rentas de cualquier naturaleza, superiores en cómputo mensual al $75 \%$ del SMI, excluida la parte proporcional de dos pagas extraordinarias, y acrediten responsabilidades familiares.

${ }^{53}$ Regulado por el Real Decreto-ley 16/2014, de 19 de diciembre, por el que se regula el Programa de Activación para el Empleo. Tendrán derecho aquellas personas que hayan permanecido inscritas como demandantes de empleo durante 270 días en los últimos 18 meses anteriores a la solicitud de la incorporación, deberán carecer de rentas de cualquier naturaleza que superen un cómputo mensual de $75 \%$ del Salario Mínimo Interprofesional -excluida la parte proporcional de dos pagas extraordinarias- y, además, puedan acreditar responsabilidades familiares.
} 
tiempo; es cierto que la pandemia sufrida a nivel mundial a partir de 2020 -y que seguimos acusando- ha propiciado que de forma urgente el Gobierno haya decidido crearla y aprobarla porque, sin lugar a dudas, se hacía necesaria una protección de urgente necesidad y que, además, se haga de forma unificada para todo el territorio nacional y que se convierta en una prestación de carácter universal dirigida a proteger a aquellos sujetos -o grupos de sujetos- susceptibles de vulneración por no poseer recursos económicos suficientes que garanticen su subsistencia y el disfrute de todos y cada uno de los derechos que nuestro ordenamiento jurídico les otorga por el mero hecho de ser personas ${ }^{54}$.

Si bien es cierto que esta prestación está orquestada en la idea básica de la universalidad hay que destacar que no es la función que realmente cumple ya que no se trata de una renta única e igual para todos y cada uno de los componentes de nuestra sociedad sino que está sesgada a partir de criterios de base económica que establecen los estándares mínimos necesarios para la obtención del derecho a la misma nota no característica de una prestación universal de carácter general ya que ello supondría que cualquier miembro de la sociedad tendría derecho a percibirla, única y exclusivamente, por el mero hecho de pertenecer a la misma independientemente de sus características personales y/o económicas; esto implica que dado el carácter restrictivo de la prestación por IMV puede darse el caso de que queden desprotegidos sujetos que de forma eventual o permanente no perciben los ingresos mínimos vitales necesarios para conseguir un estándar de vida suficiente por sobrepasar los mínimos económicos establecidos por la norma con carácter general.

Si en lugar de una prestación por IMV estuviésemos ante una renta básica universal ${ }^{55}$ caracterizada por suponer un ingreso periódico a cargo del Estado a cada uno de los ciudadanos que conforman su comunidad, convirtiéndose en un derecho sin exigencias, se podría evitar gran cantidad de situaciones defraudadoras que pueden darse en nuestro sistema de seguridad social como es el caso, por ejemplo, el poder percibir otro tipo de prestaciones independientemente de la percepción de la renta básica universal -a diferencia de lo que ocurre con el IMV que tiene establecido un régimen de

\footnotetext{
${ }^{54}$ ÁLVAREZ CORTÉS, Juan Carlos. "Una nueva prestación no contributiva de la Seguridad Social: el ingreso mínimo vital. Análisis del Real Decreto-Ley 20/2020, de 29 de mayo, por el que se establece el ingreso mínimo vital". Op. Cit., p. 285 y ss.

${ }^{55}$ La renta básica universal atiende a las necesidades de una política social cuya finalidad es redistribuir la riqueza de tal modo que se garantice un nivel mínimo de ingresos para todos los ciudadanos para, así, reducir las desigualdades existentes entre clases sociales. Dicho ingreso no está supeditado a la situación personal y/o económica del beneficiario ya que se trata de un derecho obtenido por el mero hecho de ser persona y pertenecer a una sociedad determinada en la que no se tienen en cuenta criterios tales como la situación económica, familiar o personal del solicitante si bien es cierto que, en ocasiones, sí puede afectar a la cuantía de la prestación - esta es la modalidad general y más amplia pero se cuenta con otras vertientes como, por ejemplo, sus versiones personalizadas, condicionadas al empleo o con incentivos laborales-.
} 
incompatibilidades tasado- lo que limitaría, por ejemplo, los empleos precarios o el fraude en las prestaciones del sistema. La renta básica universal sería, verdaderamente, la prestación perfecta en un mundo perfecto pero que, a día de hoy, no es más que una utopía inalcanzable ya que, obviamente, los recursos del Estado son limitados y las arcas nacionales no se encuentran en situación de poder asumir el gasto adicional que la misma supondría; conllevaría, además, la necesidad de realizar importantes reformas en materia fiscal encaminadas a la redistribución integral de la riqueza ${ }^{56}$. El IMV se ha elaborado con una base universal que, en realidad, no es tenida en cuenta ya que sin existir una situación de necesidad probada no es operativa para el individuo lo que, claramente, atiende a necesidades económicas claras y, a mi parecer, objetivas y acertadas.

La función del Estado es garantizar la subsistencia digna de todos y cada uno de los ciudadanos si bien ello conlleva que, en ocasiones, hay que ceder entre la aplicación del principio de universalidad de nuestro sistema y la búsqueda no de la igualdad sino de la equitatividad de manera que reciba más aquel que menos posee en aras de, como ya he apuntado, la subsistencia digna de los ciudadanos.

El IMV debe actuar -y actúa- sobre el compromiso constitucional para con el Estado social y democrático de derecho cuya función primordial es garantizar la dignidad de las personas y la eliminación de la desigualdad efectiva entre ciudadanos. Su función, además, es la de mejorar el sistema del Estado de bienestar a través de los principios que rigen el sistema de seguridad social, entre ellos el de solidaridad.

\section{Conclusiones}

El momento actual ha venido marcado por una dificultad económica generada, a diferencia de la anterior, por la crisis sanitaria derivada de la COVID-19; obviamente, dicha situación, ha supuesto adversidades para toda la sociedad en general si bien es cierto que puede que se haya convertido en el detonante definitivo para la aprobación de la prestación no contributiva por IMV.

La aprobación de esta nueva prestación ha supuesto el comienzo de la lucha contra la pobreza y la desigualdad entre los ciudadanos; era necesario volver a plantear, de forma actualizada, la redistribución de las rentas de los ciudadanos y prestar especial atención a la gestión de la economía focalizando los supuestos que supongan una mayor desigualdad, es decir, localizar a los colectivos más vulnerables -entre los que se encuentran, sin lugar a dudas, los niños y los jóvenes-.

\footnotetext{
56 ÁLVAREZ CORTÉS, Juan Carlos. "Una nueva prestación no contributiva de la Seguridad Social: el ingreso mínimo vital. Análisis del Real Decreto-Ley 20/2020, de 29 de mayo, por el que se establece el ingreso mínimo vital". Op. Cit., p. 285 y ss.
} 
Desde la perspectiva de los principios rectores que rigen el derecho a la seguridad social debemos tener en cuenta que la prestación no contributiva por IMV se basa en los pilares del Estado de bienestar y, por tanto, tiende a la búsqueda de la universalidad del sistema, universalidad que no puede existir de forma efectiva si no se realiza una reflexión sobre la aplicación del principio de igualdad -cabe recordar que ambos principios están íntimamente relacionados entre sí y que la consecución de uno va ligado a la aplicación efectiva del otro.

Una prestación como la que se ha tratado a lo largo de estas líneas era imprescindible y necesaria desde hace muchos años y su aprobación ha llegado en un momento complicado para la sociedad en general. La pandemia ocasionada por la COVID-19 ha forzado, finalmente, la elaboración y aprobación de la norma; como se ha indicado a lo largo de estas páginas se trata de una prestación basada en varios artículos de nuestra Constitución como, por ejemplo, el 41, el 9 y el 10.2. La protección de las personas ante situaciones de necesidad es una de las obligaciones formales que debe cumplir el Estado y esta prestación se ha convertido en una muestra de la preocupación por la consecución de los objetivos patriarcales del Estado en este sentido permitiendo ampliar de forma coherente y ampliada la protección de los individuos frente a las situaciones de necesidad.

Es cierto que la prestación por IMV no tiene el carácter universal que muchos pretendían, pero sí ha supuesto un gran paso frente a la lucha por la igualdad y la eliminación de la pobreza en nuestro país. No cabe duda de que, dentro de unos años, quizá podamos avanzar a una renta mínima universal igual para todos y cada una de las personas que componen nuestra sociedad; esta supondría un sistema universal pero no equitativo no cumpliendo con una de las finalidades primordiales de la prestación no contributiva por IMV: evitar, en la medida de lo posible, la desigualdad entre estratos sociales.

La prestación no contributiva por IMV está supeditado a las características económicas y personales de los sujetos que la soliciten y de sus beneficiarios -en forma de unidad de convivencia- y pretende proteger una situación de necesidad existente, real y latente nacida de la falta de recursos de las personas a quienes va dirigida. Su finalidad primaria es, sin lugar a dudas, garantizar la subsistencia de los individuos de manera digna que viene marcada por la obligatoriedad del Estado y por imputarse, directamente, a las arcas públicas.

La prestación por IMV permite el acceso a una determinada cantidad de renta a todas aquellas personas que ya no tienen derecho a percibir otro tipo de ingresos, prestaciones o subsidios para, así, permitir que tengan una vida digna y acceso a las necesidades básicas de las que, en ocasiones, se ve privado un alto número de miembros de nuestra sociedad. Se trata de una prestación no contributiva y, como tal, no se encuentra 
supeditada al ingreso de cotizaciones cuyo fin primordial es evitar la exclusión social y erradicar la pobreza en la medida de lo posible -si bien es el inicio de dicho camino todavía falta mucho por recorrer y conseguir.

Es cierto que clara está la postura positiva de la prestación no contributiva por IMV pero, en contraposición, aparecen las cuestiones ligadas a la vertiente negativa de la creación de dicha prestación como, por ejemplo, la posibilidad de no buscar empleo o fomento de la economía sumergida -cuestiones que se solventarían permitiendo la compatibilidad de la percepción de la prestación con otro tipo de ingresos relacionados con los trabajos realizados por cuenta ajena o propia-, es posible que, en ocasiones, la prestación no llegue a todos aquellos que realmente necesiten de la percepción de la misma por no cumplir con ciertos requisitos que para unos pueden ser fundamentales y para otros no y que, finalmente, se ha convertido en un nuevo gasto computable a las arcas de la seguridad social -si bien es cierto que estaba pensado para un gran número de personas $\mathrm{y}$, actualmente, se le ha concedido a un porcentaje aproximado al $10 \%$ del total de los previstos.

En relación a la cuestión negativa de la prestación no contributiva por IMV ha supuesto un avance inequívoco si bien es cierto que se ha establecido una serie de criterios de acceso que ha limitado la solicitud de dicha prestación. Esta cuestión atiende, sin lugar a dudas, a cuestiones puramente económicas y de sostenibilidad del sistema que, por razones obvias, hace imposible que, a día de hoy, estemos hablando de una renta mínima básica y no de una prestación no contributiva por IMV.

\section{Bibliografía}

ALARCÓN CARACUEL, Manuel Ramón. La Seguridad Social en España. Pamplona, Aranzadi, 1999, pp. 105-108.

ALONSO OLEA, Manuel, TORTUERO PLAZA, José Luis. Instituciones de Seguridad Social (18ª edición). Madrid, Civitas, 2002, p. 50.

ÁLVAREZ CORTÉS, Juan Carlos. "Una nueva prestación no contributiva de la Seguridad Social: el ingreso mínimo vital. Análisis del Real Decreto-Ley 20/2020, de 29 de mayo, por el que se establece el ingreso mínimo vital". Revista General de Derecho del Trabajo y de la Seguridad Social, no 56, 2020, pp. 285 y ss.

ARRIETA IDIAKEZ, Francisco Javier. Ingreso Mínimo Vital: compatibilidades $e$ incompatibilidades. El foro de labos [en línea]. 
(https://forodelabos.blogspot.com/2020/07/ingreso-minimo-vital-compatibilidadese.html. Consultado el 20 de agosto de 2020).

DE LA NUEZ SÁNCHEZ-CASCADO, Elisa. Ingreso mínimo vital: la regulación del Real Decreto-Ley 20/2020. Expansión [en línea]. (https://hayderecho.expansion.com/2020/06/03/ingreso-minimo-vital-la-regulacion-delreal-decreto-ley-20-2020/. Consultado el 20 de agosto de 2020).

FERNÁNDEZ MAÍLLO, Guillermo. "Ya está aquí el Ingreso Mínimo Vital”. Papeles de relaciones ecosociales y cambio global, $\mathrm{n}^{\mathrm{o}}$ 150, 2020, pp. 83-92.

GARCÍA DÍAZ, Miguel Ángel. "La seguridad social en España: Situación actual y algunas reflexiones sobre su futuro". Cuadernos De Relaciones Laborales, (8), 1996, pp. 85-105.

HERRANZ, Cristina. Guía básica del Ingreso Mínimo Vital (IMV). Economist \& Jurist [en línea]. (https://www.economistjurist.es/noticias-juridicas/guia-basica-del-ingresominimo-vital-imv/. Consultado el 10 de agosto de 2020).

JIMENA QUESADA, Luis. "El derecho a la protección contra la pobreza y la exclusión social como paradigma del respeto de la dignidad humana. La inserción del ingreso mínimo vital en el marco de la evolución de los estándares internacionales". Lex Social: Revista de Derechos Sociales, vol. 10, n² 2, 2020, pp. 361-423.

MALAVIA, Miguel Ángel. "Ingreso Mínimo Vital: un paso contra las colas del hambre". Vida Nueva, no 3182, 2020, pp. 8-13.

MONEREO PÉREZ, Jose Luis, MOLINA NAVARRETE, Cristóbal, QUESADA SEGURA, Rosa. Manual de Seguridad Social (11 a edición). Madrid, Tecnos, 2015, p. 90.

MONEREO PÉREZ, Jose Luis. "La renta de "subsistencia". En especial, la renta de inserción como derecho social". En La jurisprudencia constitucional en materia laboral y social en el período 1999-2010: libro homenaje a María Emilia Casas. La Ley (España), 2015. pp. 713-755.

MONEREO PÉREZ, Jose Luis. "Nuevos derechos para nuevas situaciones de necesidad y de emergencia social: el ingreso mínimo vital". Estudios financieros. Revista de trabajo y seguridad social: Comentarios, casos prácticos: recursos humanos, $\mathrm{n}^{\circ} 448,2020$, pp. 27-68. 
RODRÍGUEZ RAMOS, María José, GORELLI HERNÁNDEZ, Juan Marco, VÍLCHEZ PORRAS, Antonio. Sistema de Seguridad Social (7 edición). Madrid, Tecnos, 2005, p. 71.

SALA FRANCO, Tomás, MARTÍN-POZUELO LÓPEZ, Ángela. El Ingreso Mínimo Vital. El sistema español de rentas mínimas. Valencia, Tirant Lo Blanch, 2020. 